\title{
Clinically Studies on Treatment of Pulmonary Arterial Hypertension Induced Right Heart Failure
}

\author{
Yudong Wang* \\ The Central Hospital of Zhumadian City, Zhumadian, Henan 463000, China
}

\begin{abstract}
Objective: to study a clinical treatment of pulmonary arterial hypertension induced right heart failure. Method: A total of 60 cases of pulmonary heart disease right heart failure patients were randomly divided into observation group and control group in which 30 cases for each group. Results: In the observation group of 30 cases, 14 cases produce an effect, 15 cases is effective, 2 cases is invalid and the total effective rate is $96.6 \%$. In the control group of 30 cases, 8 cases produce an effect, 17 cases is effective, 5 cases is invalid and the total effective rate is $83.3 \%$. Conclusion: Dobutamine can alleviate symptoms of heart failure, reduce heart failure patients' fatality rate and improve prognosis.
\end{abstract}

\author{
KEYWORDS \\ Pulmonary arterial \\ Hypertension \\ Dobutamine \\ Heart failure
}

\section{Introduction}

Pulmonary Arterial Hypertension (PAH) refers to a kind of pathophysiology syndrome where weakened of vascular caused by any system or local lesion which make the whole pulmonary pressure increased, eventually leads to right heart failure. This study aims to discuss about the right arterial hypertenion leads to heart failure as the research object and to explore the diagnosis and treatment of right heart failure.

\section{Materials and method}

\subsection{General information}

A total of 60 patients with right heart failure were randomly choose including male 40 cases, female 20 cases, aged 55-70 years old, with an average age of 60.5 years. All the subjects were choose in accordance with the standards which are consistent with 2008 Haddad of right heart failure clinical staging criteria 2: (1) Period with right heart failure risk factors have no heart failure symptoms; (2) period with right ventricular dysfunction or organic heart disease no heart failure symptoms; (3) Period with right

Copyright (๑) 2015 Yudong Wang

doi: $10.18686 /$ aem.v4i1.7

Received: December 29, 2014; Accepted: February 14, 2015; Published online: March 28, 2015

This is an open-access article distributed under the terms of the Creative Commons Attribution Unported License (http://creativecommons.org/ licenses/by-nc/4.0/), which permits unrestricted use, distribution, and reproduction in any medium, provided the original work is properly cited.

${ }^{\star}$ Corresponding author: The Central Hospital of Zhumadian City, Zhumadian, Henan 463000, China. E-mail: wang_yd535@sina.com ventricular dysfunction or structural heart disease; (4) Period refractory right heart failure. All patients were randomly divided into observation group and control group where 30 cases of each group, two groups of gender, age and course of the disease. The clinical data comparison difference show that there has no statistical significance $(p>$ $0.05)$, have comparability.

\subsection{Treatment}

In control group 30 cases of patients were applied with loop diuretic torasemide for first dose of $20 \mathrm{mg}$ through intravenous injection and after 2-4 hours later dose is reduced to half; $0.4 \mathrm{mg}$ of Cardiac glycosides deacetylation lanatoside was injected via intravenous injection, with every 2-4 hours later give $0.2-0.4 \mathrm{mg}$ and total was $1-1.6$ $\mathrm{mg}$; Based on the ventricular rate adjust the dosage, after patients with clinical symptoms stabled and fluid retention marked improved, switch to digitalis preparations (start with small doses, until the maximum tolerated dose).

On the other hand, observe group on the basis of control group were used positive inotropic drug dobutamine $200 \mathrm{mg}$ of $50 \mathrm{ml}$ dubbed into micro pump, started with small dose $1-5 \mathrm{ug} / \mathrm{kg} / \mathrm{min}$ by center vein pipe pump, and increase the dosage until until reach $8-12 \mathrm{ug} / \mathrm{kg} / \mathrm{min}$. Patients with severe hypotension patients can be associated with dopamine or milrinone.

\subsection{Curative effect judgment standard}

Marked clinical criteria: improvement of cardiac class II or above; (1) Effective: Heart function improved class I or above, Part of symptoms remitted and signs were reduced. 
Table 1. Comparison of two groups after treatment effect, confinement (day) and total effective rate (\%).

\begin{tabular}{ccccccc}
\hline Group & $\mathrm{n}$ & Produce an effect & Effective & Invalid & Confinement (day) & Total effective rate (\%) \\
\hline Observe group & 30 & 14 & 15 & 1 & $7 \pm 3$ & 96.6 \\
Control group & 30 & 8 & 17 & 5 & $10 \pm 4$ & 83.3 \\
\hline
\end{tabular}

(2) Invalid: heart function improved less than class I, signs and symptoms not improved, even become worse.

\subsection{Statistical method}

Two groups were compared by $t$ test and the total efficiency was expressed in percentage (\%) of each group and the number of cases was compared by $r$ test. The following table is as Table 1.

All the data were analyzed by SPSS 15.0 statistical software when $p<0.05$, the difference was statistically significant.

\section{Result}

In the observation group of 30 cases, 14 cases produce an effect, 15 cases is effective, and 2 cases is invalid where the total effective rate is $96.6 \%$. In the control group of 30 cases, 8 cases produce an effect, 17 cases is effective and 5 cases is invalid, the total effective rate is $83.3 \%$. Two group total effective rate and confinement (day) have significant difference $(p<0.05)$.

Two groups of patients all have no adverse events occurred during the use of medication. There is no abnormal in blood, urine, stool routine and liver and kidney function during the period of medication. Thus, we can see safety of the drug is good and does not appear obvious side effects.

\section{Discussion}

Pulmonary arterial hypertension is pulmonary vascular bed exhausted caused by a variety of causes, make progressive pulmonary resistance increases, eventually leads to right heart failure pathophysiology syndrome. Pulmonary arterial hypertension is the main reason of right heart failure, and right heart failure is the only way to cause all types of pulmonary hypertension patient disability and death. Not only that, it is the most important factors of pulmonary arterial hypertension patient prognosis. At present, local and international guidelines will reflect the right parameters are indicators of cardiac function in patients with pulmonary hypertension as important risk stratification. However, a related function of right ventricle is still a relatively new field. Therefore, the US National Heart, Lung and Blood Institute will be listed as the focus of future research direction in the field of cardiovascular. In recent years, pulmonary arterial hypertension induced right heart failure gradually be taken seriously, there is likely to become one of the hotspot in research of heart failure in the future [1].

Pulmonary arterial hypertension induced right heart failure has been a research focus in recent years. The early pulmonary arterial hypertension can lead to the right ven- tricle compensatory reconstruction, but still can maintain normal cardiac output and against the increase of pulmonary vascular resistance against the formation of right ventricular hypertrophy. As the progress of the disease, the right ventricle hypertrophy insufficient against pressure load change beyond the scope of myocardial compensatory, thus decompensated ventricular reconstruction, that is the right heart failure. In the pulmonary arterial hypertension induced by chronic right heart failure model, heart chamber become plump due to compensatory, then happen the right heart failure, pulmonary arterial hypertension induced disease throughout the right heart. Research shows that when pulmonary hypertension patients in right ventricular filling the biggest, the right ventricle through a ventricular septal to left ventricle there is a pressure gradient. This is because the right ventricular pressure overload lead to right ventricular free wall contraction prolonged. However, as left ventricle has entered the early diastolic, right ventricular pressure has more than the left ventricle, leads to the formation of a cross between pressure gradient, and appear ventricular septal reverse movement. This not only affect the right ventricular end systolic contraction, also reduces the early left ventricular diastolic filling, make left ventricular end-diastolic volume reduced and directly affects the left ventricular cardiac output, in turn lead to pulmonary pressure rise further. This will promote the occurred and development of right heart failure. Pulmonary arterial hypertension leads to right heart failure has many other factors, such as: ventricular remodeling, neural endocrine hormone, cytokine activation, myocardial cell apoptosis, inflammation, oxidative stress and abnormal gene expression, etc, they through the pulmonary hypertension, and lead to right heart failure occurred during the process of development.

General treatments of right heart failure include control fluid retention, limiting sodium intake ( $\leq 2 \mathrm{~g} / \mathrm{d})$, body weight monitoring, careful application of diuretics. Classification of physical activity in patients with right heart failure and pulmonary hypertension may be beneficial. Recent studies have shown that patients with chronic severe pulmonary hypertension have moderate intensity exercise can significantly improve the sport ability and life quality. There are many clinical trials have confirmed that $\beta$-receptor blockers can improve the symptoms of heart failure and the cardiac function and thus reduce death. Angiotensin II converting angiotensin enzyme inhibitor (ACEI) and angiotensin II receptor blocker (ARB) which can lower blood pressure and cardiac afterload thus reduce the thickness and stiffness of the arterial wall, weak angio- 
tensin II will effect on the contraction of coronary artery thus improve cardiac function. Since most of right heart failure is associated with pulmonary hypertension, in view of the targeted drug therapy pulmonary hypertension can progress soon, mainly include calcium channel blockers, prostacyclin and its structural analogs, endothelin receptor antagonist, phosphodiesterase type 5 inhibitors and Rho kinase inhibitors. China scholars have confirmed, sildenafil can safely and effectively treat pulmonary hypertension, and recommended to use sildenafil $25 \mathrm{mg}, 3$ times a day. In addition, Rho kinase inhibitors may be a promising selective pulmonary artery vasodilators, but still need further clinical trials [2].

The right ventricle structure anatomical and functional features have great differences with left ventricular where right ventricular myocardial fibers are arranged in series, compared with the left ventricle. The characteristics of the right ventricle are wall thin, low pressure, small volume and surface area ratio is small, so it have good compliance. Therefore if the right ventricle load is increased the heart chamber will eventually expand to accommodate the increased of pulmonary artery pressure. The pathogenesis of right heart failure is very complicated and maybe include myocardial injury and stress, sympathetic nervous system (SNS), renin-angiotensin aldosterone system (RAAS), activation of endothelin (ET) and so on ventricular remodeling, etc. Heart failure also activate neurotrophic factor like: vasopressin, prostaglandins, atrial peptide, brain natriuretic peptide, tumor necrosis factor, interleukin-1, interleukin- 6 and other neurological factors. These neuroendocrine factor activation levels and severity of heart failure were positively correlated. Neuroendocrine system activation can be directly toxic to the heart and activate vascular endothelial directly, make myocardial cell thickening, apoptosis and necrosis, increase myocardial fibrosis and make heart expending and reshaping. Heart reshaping lead to heart structure and functional change is the mechanism of heart failure where the excessive activation of neuroendocrine system plays a very important part in heart reshaping. Pulmonary arterial hypertension is the important determinants in right heart function for failure development process. In the process of the development of PAH, molecular and genetic levels factors exist in smooth muscle endothelial cells and vascular adventitia, also exist constriction of blood vessels and vasodilation environmental imbalances, those all are pulmonary hypertension research directions. Pulmonary hypertension and right heart failure play an important role, in order to overcome the resistance of pulmonary hypertension and right ventricular hypertrophy [3]. When early pulmonary hypertension occur, right ventricular still capable for compensation, however when late pulmonary artery pressure continues to rise, more compensatory ability is required for the right ventricle. This will lead to right heart failure where decreased cardiac output, right ventricular end-systolic residual blood volume increased as well as end-diastolic pressure increased. Right heart failure includes pressure overload, volume overload, myocardial ischemia, myocardial disease and pericardial diseases. Based on anatomy of right and left ventricles, different features of right heart failure treatment cannot be equated in left heart failure treatment. After the 1990s, gradually clear cardiac remodeling is a fundamental mechanism for the development of heart failure and neural endocrine suppression blocking cardiac remodeling. Failure to repair the biological properties of cardiomyocytes is a milestone in the history of the treatment of heart failure. Right heart failure in patients with acute hemodynamic disorders often uses inotropic drugs as a treatment. This study was a joint group of inotropic dobutamine, to adjust cardiac function through controlled observation and the application in patients with heart failure remission after amine level, the total effective rate, there was a significant difference in hospitalization date with the control group. Therefore, dobutamine is able to improve cardiac index, increase cardiac output, reduce pulmonary arterial resistance and relieve symptoms of heart failure, reduced mortality and improve the prognosis of patients. After all, this treatment is worthy for clinical application

\section{References}

1. Norbert FV, Robert AQ, Leslie AL, et.al. Right ventricular function and failure: Report of a national, lung, and blood institute working group on cellular and molecular mechanisms of right heart failure. Circulation. 2006;14(1):183-189.

2. Wang YZ. Chronic heart failure clinical efficacy of captopril. China Modern Drug Application. 2009,3(13):119-120.

3. LU YX. Theoretical basis evolution of the concept of treatment of heart failure. Clinical Internal Medicine. 2004;21:217-219. 